\title{
Some observational manifestations of the periodic inhomogeneity of absorbing and scattering atmospheres
}

\author{
A. Nikoghossian* \\ NAS RA V.Ambartsumian Byurakan Astrophysical Observatory, Byurakan 0213, Aragatsotn Province, Armenia
}

\begin{abstract}
We consider frequency and directional features of the reflectance and transmittance of an inhomogeneous scattering and absorbing atmosphere with the scattering albedo periodically varying with depth. The dependence of global optical properties of such media on optical thickness is found. Two different kind of problems are considered dependent on that by monochromatic or continuum, mono-directional or diffuse radiation illuminate the medium. The numerical results show how the observed intensities and line-profiles respond to the medium inhomogeneity with an uniform increase in optical thickness. Astrophysical aspects of these features are discussed.
\end{abstract}

\section{Introduction}

It is well established that at some stages of their evolution the stars exhibit the matter outflow in the form of a more or less dense wind forming extended envelops. Luminous Blue Variables (LBVs), $\mathrm{Ae}, \mathrm{Be} \mathrm{He}$ and T Tau stars are good examples of this kind intensively studied astronomical objects. Especially exotic are luminosity variations of LBV stars which may change their spectral class under almost constant value of bolometric luminosity. As it was shown by (Israelian \& Nikoghossian, 1996, Nikoghossian \& Israelian, 1996), this effect can be explained by appearing of some centers coherently scattering in the continuum (e.g., free electrons) leading to an increase in optical thickness of winds.

The temporal irregularity of the luminosity variation is usually interpreted by changes in the temps of outflow and other non-stationary phenomena as the matter accretion from companion stars. It must be, however, noted that in the majority of cases the ejected matter is assumed homogeneous which hardly is realisable in the reality. This insists on considering the general case of inhomogeneous envelopes with irregular variations of global thermodynamic parameters which determine the radiation scattering process in the medium.

The paper may be considered as an attempt to fill this gap by revealing how and at what extent observational characteristics mimic the changes in physical papameters inside the radiating medium.

\section{Formulation of the problem and basic equations.}

For simplicity's sake, we consider the ID frequency-dependent and 3D mono-chromatic problems of the line-radiation transfer in scattering and absorbing media of different optical thicknesses. The inhomogeneity of the medium is supposed to be due to that of the scattering coefficient (particle albedo or the probability of re-radiation in the elementary act of scattering denoting by $\lambda$ ) changes within the medium according to a certain periodical law of the form $\lambda(\tau)=0.5(1+\sin 3 \tau)$. This choice of the function $\lambda(\tau)$ is due to easier tracking the observational effect of changes in the physical state in the medium. We limit ourselves by treating periodically varying inhomogeneity, however, its results evidently allow to make an idea on effects expected in the case of any irregular variation of this parameter.

\footnotetext{
*nikoghoss@yahoo.com
} 
Thus, we are interested in the behavior of the reflectance of the medium from the side of $\tau=\tau_{0}$ and its transmittance with increasing the optical thickness $\tau_{0}$.

We begin with treating the ID problems of the frequency-dependent radiation transfer for different values of optical thickness. We limit ourselves with considering the case of complete redistribution of radiation over frequencies. Two cases are of interest: the medium is illuminated by monochromatic radiation of a given frequency and it illuminated by radiation of unit intensity in the continuum.

The basic equations for determination of the reflectance $r\left(x^{\prime}, x\right)$ and transmittance $q\left(x^{\prime}, x\right)$ are obtained with use of invariant imbedding approach (see,for instance, Ambartsumian (1944), Ambartsumyan (1960), Bellman et al. (1960), Chandrasekhar (1960), Nikoghossian (2012), Sobolev (1963)). In our case the coefficient $r$ determines the reflectance of the medium from the side of the boundary $\tau_{0}$ dependent on frequencies $x^{\prime}, x$ of incident and reflected quanta (as usual, we deal with dimensionless frequencies, defined as the displacement from the line center measured in Doppler widths).

We have

$$
\frac{d r}{d \tau_{0}}=-\left[\alpha(x)+\alpha\left(x^{\prime}\right)\right] r\left(x^{\prime}, x, \tau_{0}\right)+\frac{\tilde{\lambda}\left(\tau_{0}\right)}{2} \varphi\left(x, \tau_{0}\right) \varphi\left(x^{\prime}, \tau_{0}\right)
$$

where $\alpha$ is the profile of the absorption coefficient and

$$
\varphi\left(x, \tau_{0}\right)=\alpha(x)+\int_{-\infty}^{\infty} r\left(x, x^{\prime}, \tau_{0}\right) \alpha\left(x^{\prime}\right) d x^{\prime}
$$

Equation (1) satisfies the physically evident initial condition $r\left(x^{\prime}, x, 0\right)=0$.

Turning to the transmittance $q$, we separate its direct and diffuse parts as follows

$$
q\left(x^{\prime}, x, \tau_{0}\right)=\delta\left(x-x^{\prime}\right) \exp \left[-\alpha(x) \tau_{0}\right]+\sigma\left(x^{\prime}, x, \tau_{0}\right),
$$

where $\delta$ is the Dirac $\delta$-function. Being applied the invariant imbedding procedure leads to integraldifferential equation for the function $\sigma\left(x^{\prime} x, \tau_{0}\right)$

$$
\frac{d \sigma}{d \tau_{0}}=-\alpha(x) \sigma\left(x^{\prime}, x, \tau_{0}\right)+\frac{\tilde{\lambda}\left(\tau_{0}\right)}{2} \varphi\left(x, \tau_{0}\right) \psi\left(x^{\prime}, \tau_{0}\right)
$$

with an initial condition $\sigma\left(x^{\prime}, x, 0\right)=0$. The function $\psi$ appeared in Eq.(3) is given by the formula

$$
\psi\left(x^{\prime}, \tau_{0}\right)=\int_{-\infty}^{\infty} q\left(x^{\prime}, x^{\prime \prime}, \tau_{0}\right) \alpha\left(x^{\prime \prime}\right) d x^{\prime \prime} .
$$

Analogous integral-differential equation can be easily derived also for the reflectance of the medium from the side of the boundary $\tau=0$. This quantity, however, is not of interest by its physical content in this particular case of inhomogeneity, especially for large optical thicknesses.

Let's turn now to analogous equations obtained by the same way for the 3D transfer problem where of primary interest for us is the spatial distribution of the reflected and transmitted radiation. Consider the diffuse reflection and transmission of monochromatic radiation for a plane-parallel finite atmosphere assuming, for simplicity, that the scattering is isotropic.

Following the notations adopted in Nikoghossian (2012), we introduce the reflection coefficient for the boundary $\tau_{0}, \rho\left(\eta, \xi, \tau_{0}\right)$, where $\xi$ is cosine of the angle of incidence and $\eta$ is cosine of the reflection angle (the angles are counted from direction of the outward normal). It is introduced in such a way that the quantity $\rho\left(\eta, \xi, \tau_{0}\right) / \xi$ has a probabilistic meaning.

The function $\rho\left(\eta, \xi, \tau_{0}\right)$ satisfies the equation (see, Chandrasekhar (1960), Sobolev (1963))

$$
\frac{d \rho}{d \tau_{0}}=-\left(\frac{1}{\eta}+\frac{1}{\xi}\right) \rho\left(\eta, \xi, \tau_{0}\right)+\frac{\lambda\left(\tau_{0}\right)}{2} \varphi\left(\eta, \tau_{0}\right) \varphi\left(\xi, \tau_{0}\right),
$$

where

$$
\varphi\left(\eta, \tau_{0}\right)=1+\int_{-\infty}^{\infty} \rho\left(\eta, \eta^{\prime}, \tau_{0}\right) \frac{d \eta^{\prime}}{\eta^{\prime}} .
$$



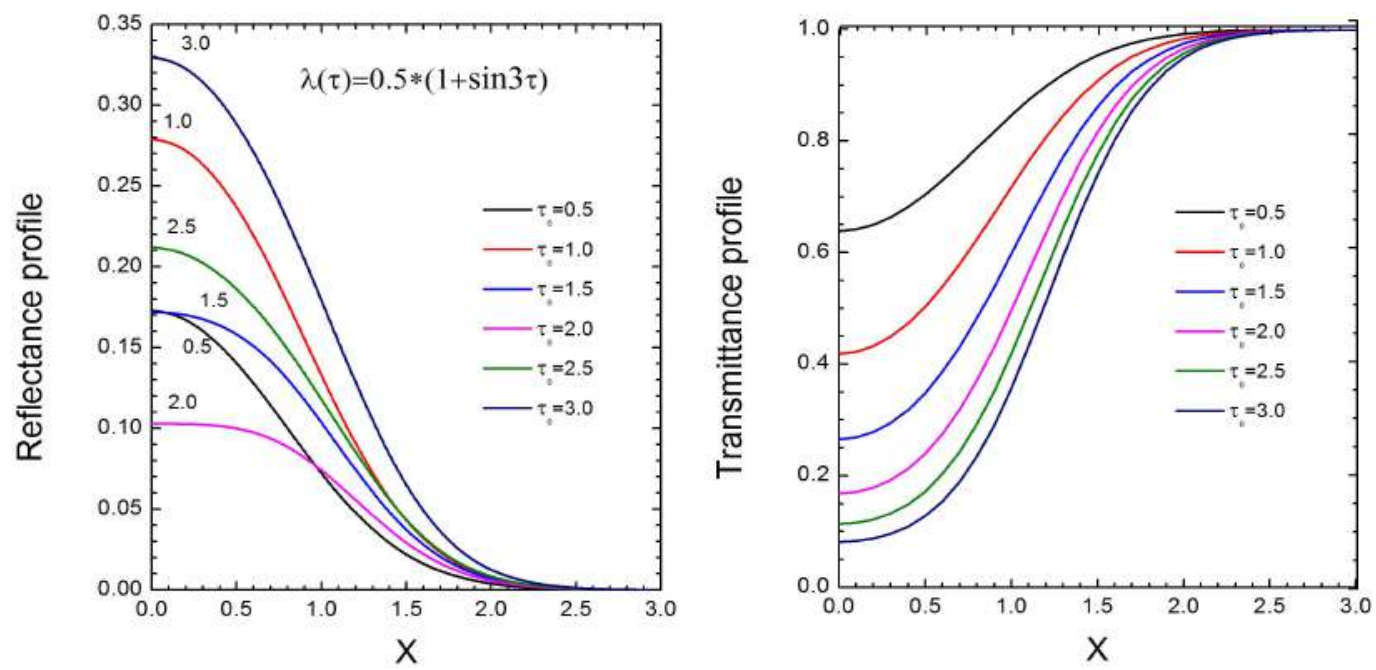

Figure 1. Reflected and transmitted lines profiles for media of different optical thicknesses illuminated by radiation in continuum.

with $\rho(\eta, \xi, 0)=0$

Separating again the direct and diffuse parts in the transmission coefficient

$$
q\left(\eta, \xi, \tau_{0}\right)=\xi \delta(\eta-\xi) \exp \left[-\tau_{0} / \xi\right]+\sigma\left(\eta, \xi, \tau_{0}\right),
$$

we arrive at the following differential-integral equation for the function $\sigma$

$$
\frac{d \sigma}{d \tau_{0}}=-\frac{1}{\xi} \sigma\left(\eta, \xi, \tau_{0}\right)+\frac{\lambda\left(\tau_{0}\right)}{2} \psi\left(\eta, \tau_{0}\right) \varphi\left(\xi, \tau_{0}\right),
$$

where

$$
\psi\left(\eta, \tau_{0}\right)=\int_{0}^{1} q\left(\eta, \eta^{\prime}, \tau_{0}\right) \frac{d \eta^{\prime}}{\eta^{\prime}}=\exp \left(-\frac{\tau_{0}}{\eta}\right)+\int_{0}^{1} \sigma\left(\eta, \eta^{\prime}, \tau_{0}\right) \frac{d \eta^{\prime}}{\eta^{\prime}}
$$

The initial condition obviously is $\sigma(\eta, \xi, 0)=0$

\section{Numerical results.}

We begin with results concerned the 1D frequency-dependent problem for the case when the boundary $\tau_{0}$ of finite medium is illuminated by the continuum radiation of unit intensity. Fig 1 shows the evolution of observed reflected and transmitted profiles with increasing optical thickness. It is striking variations in the reflected profiles which change in unison with the changes in the scattering level within the medium. The oscillations remain discernable even for pretty thick media $\left(\tau_{0} \approx 3\right)$. This effect is of interest in the sense that oscillations in the observed profiles are not related with any temporary changes in physical properties of the scattering and absorbing medium. Here the effect is due to inhomogeneity of the medium and to continuously varying opacity which in reality can be imagined as a stationary outflow of the matter from a star. The effect of inhomogeneity on transmitted profiles (right panel) is not so remarkable especially for high values of the optical thickness, which is tractable on the physical grounds.

Graphs of numerical results for monochromatic problem are depicted on Fig.2. We limit ourselves by demonstrating observed profiles only for the case when the medium is illuminated by monochromatic radiation in the center of the line. We see that the effect in the reflected profiles are similar to those discussed above.

Significant impact is discernable in transmitted emission profiles. The profiles response to the medium inhomogeneity is now both quantitative and qualitative. It is striking the appearance of 

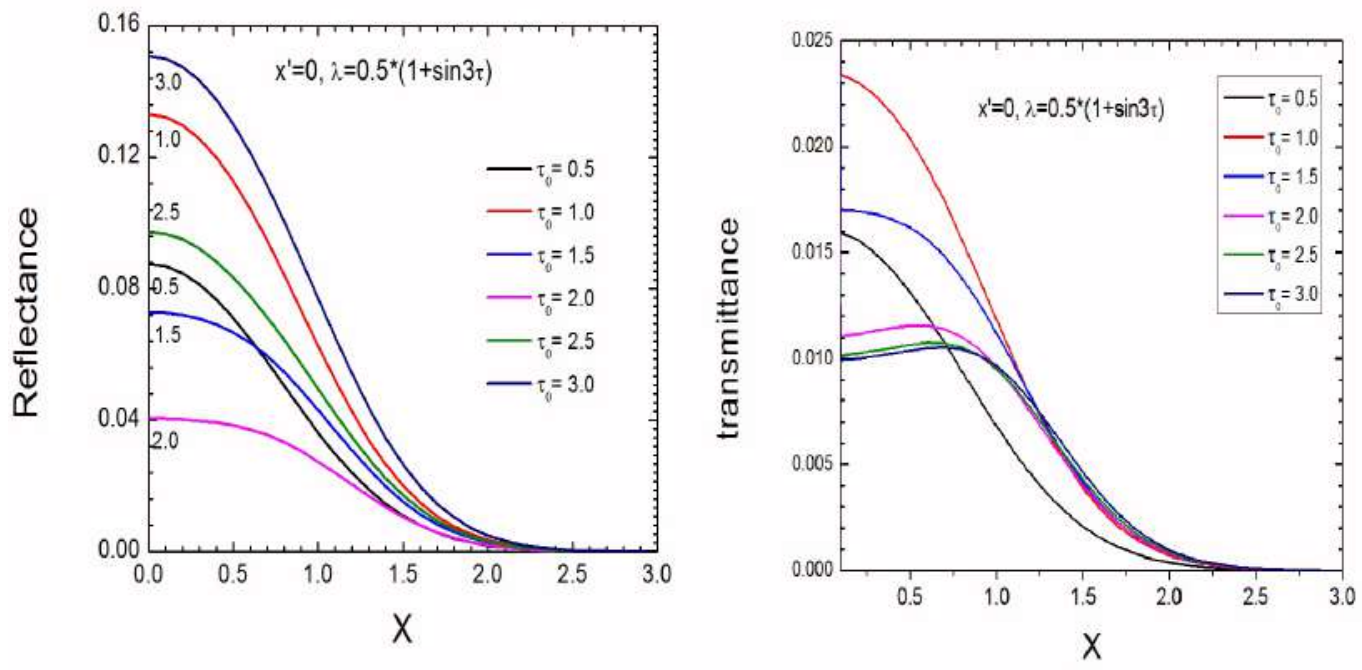

Figure 2. Reflected and transmitted lines profiles for media of different optical thickness illuminated by mono-chromatic radiation in the center of the line.

double-picked profiles. Now oscillations are observable not only in reflected but also in transmitted profiles. It is reasonable to expect such pictures are feasible in the cases of any kind irregular variations in physical properties of scattering medium.

Let us turn now to Figs.3, 4 and follow the spatial effect of scattering inhomogeneity. Fig. 3 exhibits the spatial distribution of the reflected and transmitted radiation for the case of monodirectional illumination of the boundary $\tau_{0}$. It is supposed that the radiation falls in direction of the normal to the surface of the medium. Being plotted in polar coordinates the curves show the nature of aperture oscillations of observed radiations. We see that the effect is significant for both the reflected and transmitted radiation.

Similar calculations were performed assuming that the medium is illuminated by diffuse radiation of unit intensity. The results are depicted in Fig.4. Plots in Cartesian coordinates allow to better trace spatial oscillations of observed radiation with an increase of the optical thickness. it is noticeable that the character of the observed radiation changes depending on the angle of deflection from the normal direction is different for opaque and optically thin media.

\section{Discussion}

The paper may be regarded as a first attempt to find out the response of the observed line-radiation to inhomogeneity of the scattering and absorbing medium. We exemplify it by taking the scattering coefficient as a depth-varying characteristics of inhomogeneous medium. The periodic functional form of the law of its variation was chosen for reasons of clarity of the observed effect and, in principle, can be replaced by any other law describing irregular variations in physical characteristics of the medium. The importance of obtained results is due to the fact that, as we saw, the oscillatory radiation is not necessarily due to any kind of non-stationary phenomenon, but may be caused by some specific variations of physical conditions in the medium. We have seen that significant oscillations are subject to both reflected and transmitted fluxes. Astrophysical aspect of this problem is a matter of separate investigation involving radiation scattering in the continuum 


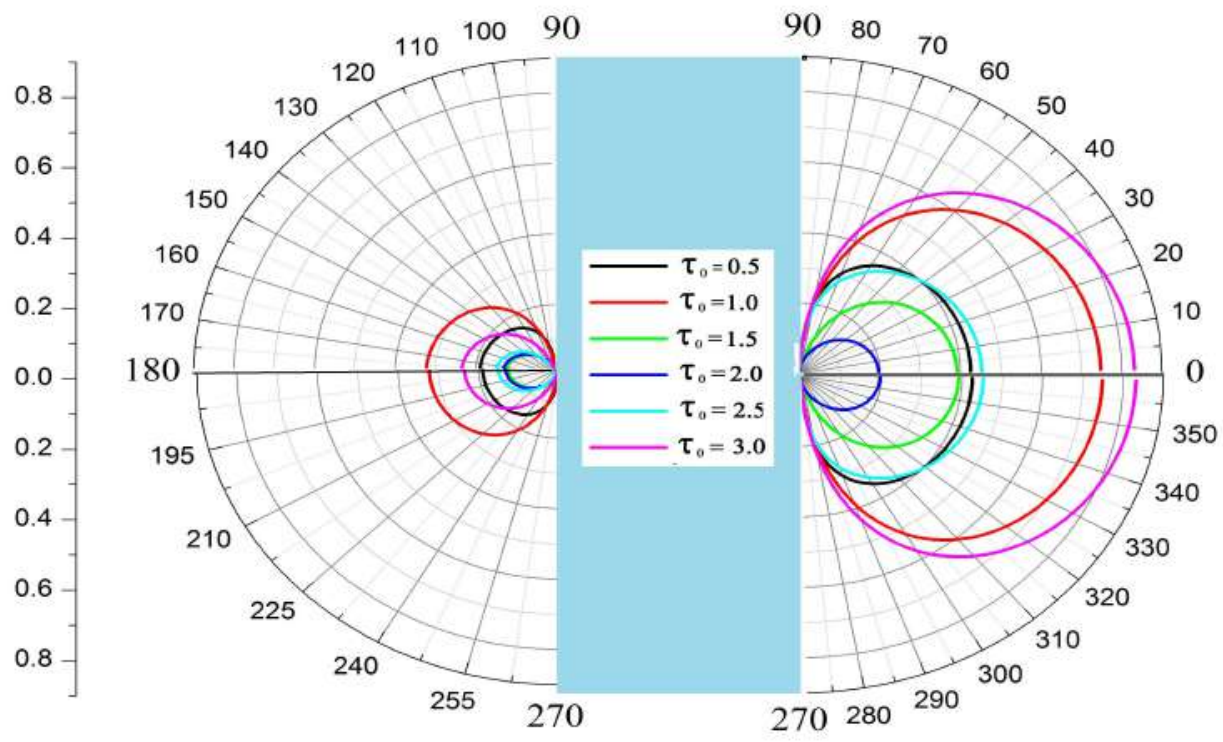

Figure 3. Spatial distribution of reflected and transmitted radiation for media illuminated by diffuse radiation.
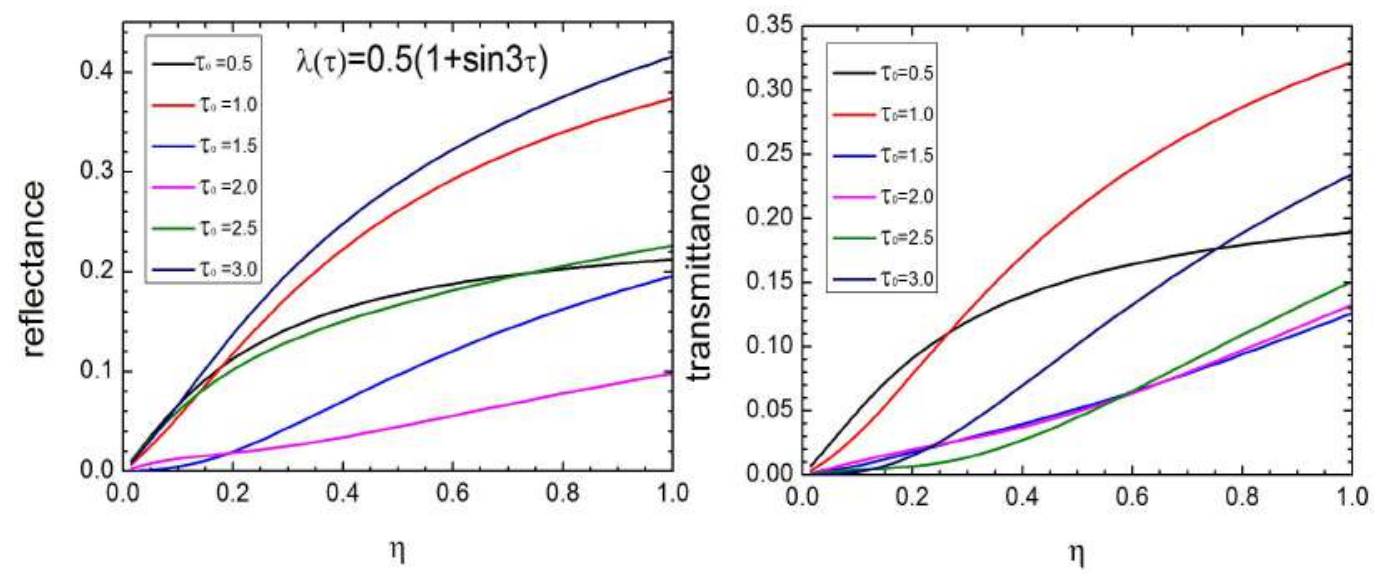

Figure 4. Spatial distribution of reflected and transmitted radiation for media illuminated by monodirectional radiation in direction of the normal to the surface. 


\section{References}

Ambartsumian V. A., 1944, Izv. Akad. Nauk ArmSSR, N 1, 2

Ambartsumyan V. A., 1960, Scientific Works, V. I, Yerevan,Izd. Acad. Nauk ArmSSR

Bellman R., Kalaba R., Wing G. M., 1960, Journal of Mathematical Physics, 1, 280

Chandrasekhar S., 1960, Radiative transfer

Israelian G., Nikoghossian A., 1996, JQSRT, 56, 509

Nikoghossian A. G., 2012, Astrophysics, 55, 261

Nikoghossian A., Israelian G., 1996, JQSRT, 56, 501

Sobolev V. V., 1963, A Treatise on Radiative Transfer, Princeton: van Nostrand., 